\title{
Archéopages
}

Archéopages

Archéologie et société

43 | 2016

Médecines

\section{Exploitation et utilisation des invertébrés marins durant la Protohistoire sur le territoire continental et littoral Manche-Atlantique français}

\section{Caroline Mougne}

\section{CpenEdition}

Journals

Édition électronique

URL : https://journals.openedition.org/archeopages/900

DOI : $10.4000 /$ archeopages.900

ISSN : 2269-9872

Éditeur

INRAP - Institut national de recherches archéologiques préventives

Édition imprimée

Date de publication : 1 novembre 2016

Pagination : 108-109

ISSN : 1622-8545

\section{Référence électronique}

Caroline Mougne, «Exploitation et utilisation des invertébrés marins durant la Protohistoire sur le territoire continental et littoral Manche-Atlantique français », Archéopages [En ligne], 43 | 2016, mis en ligne le 01 décembre 2018, consulté le 03 juin 2021. URL : http://journals.openedition.org/ archeopages/900; DOI : https://doi.org/10.4000/archeopages.900 


Un FÉVRIER 2015
Université de Rennes 1
Directeurs de thèse
José Gomez de Soto
Directeur de recherche émérite, CNRS
Catherine Dupont
Chargée de recherche, CNRS
Membres du jury
Président
Jean-Denis Vigne
Directeur de recherche, CNRS
Rapporteurs
Daniella E. Bar-Yosef Mayer,
chargée de recherche,
université de Tel Aviv
Patrice Méniel,
directeur de recherche, CNRS
Examinateurs
Marie-Yvane Daire,
directeur de recherche, CNRS
Claude Mordant,
professeur émérite, université
de Bourgogne
Consultable
sous format papier à l'université
de Rennes 1 . Service de la
documentation du CReAAH/CNRS.
Meche

Caroline Mougne

UMR 6566, « CReAAH »

\section{Exploitation et utilisation des invertébrés marins durant la Protohistoire sur le territoire continental et littoral Manche-Atlantique français}

Cette thèse porte sur l'exploitation et l'utilisation des invertébrés marins (mollusques, crustacés et échinodermes) durant l'âge du Bronze et l'âge du Fer dans l'Ouest de la France. L'objectif de ce travail est d'appréhender les relations qui unissaient les communautés protohistoriques du littoral MancheAtlantique français. Les recherches s'appuient sur l'inventaire de 197 sites ayant livré des restes d'invertébrés marins et sur les études archéomalacologiques détaillées de 32 sites, dont 17 réalisées par nos soins. Dans le cadre des problématiques abordées dans cette recherche, plusieurs approches méthodologiques novatrices, voire inédites, ont été développées. La majorité porte sur la reconstitution de tailles originelles des invertébrés marins à partir de fragments afin d'acquérir des résultats sur les techniques de collecte et, plus largement, sur les pratiques économiques. Trois espèces ont fait l'objet de ce type de reconstitution, à savoir la moule commune Mytilus edulis, l'oursin violet Paracentrotus lividus et le crabe sillonné Xantho sp. Ces reconstitutions ont été réalisées à partir de fragments de coquilles pour la moule, des parties dures pour l'oursin et d'une zone de la pince pour le crabe. Les coefficients de corrélation avoisinent les 0,9 et permettent une reconstitution fiable. Ces méthodes ont ainsi contribué grandement à l'enrichissement et à la fiabilité des données cidessous exposées.

Les résultats obtenus concernent l'exploitation des milieux, les pratiques alimentaires, artisanales, architecturales, funéraires et cultuelles des populations protohistoriques concernées. Les environnements littoraux exploités dans l'Ouest de la France durant lâge du Bronze et l'âge du Fer sont essentiellement les milieux rocheux. Ce type de substrat a pu être choisi du fait d'une plus grande accessibilité aux espèces qui y vivent, dans la mesure où ces dernières peuvent être repérées directement à la surface du rocher. L'exploitation du milieu sableux semble, quant à elle, fortement liée à des contextes funéraires et cultuels et à des utilisations singulières (parure et dépôt). La totalité des espèces consommées présentes sur les sites a pu être collectée à pied sec, en zone intertidale. L'environnement proche d'un site et les invertébrés marins disponibles localement ont probablement joué un rôle important dans les choix des espèces consommées, indiquant la pratique d'une collecte à pied réalisée dans les environs immédiats de l'habitat. Toutefois, le spectre des espèces découvertes sur les sites archéologiques révèle généralement une collecte sélective non représentative de l'ensemble de la variété disponible sur place, ce qui suggère des choix culturels.

En effet, l'étude des pratiques alimentaires a souligné des spécificités régionales. La Basse-Normandie, la Bretagne et le Poitou-Charentes se caractérisent ainsi par des assemblages malacologiques et un mode de sélection distincts. En Bretagne, la patelle Patella sp. est omniprésente. Cependant, cette dernière est totalement absente de l'alimentation des habitants de la Basse-Normandie, qui consomment essentiellement des moules communes. Pour ce qui est du Poitou-Charentes, le spectre est différent selon les sites, même pour ceux de période identique. Il est à noter que les populations protohistoriques de cette région ne semblent pas s'être focalisées sur le ramassage d'une seule espèce, à l'inverse des régions plus septentrionales.

D'un point de vue diachronique, en Basse-Normandie et en Bretagne, les coquillages marins sélectionnés semblent identiques pendant lâge du Bronze et l'âge du Fer. Inversement, en Poitou-Charentes, une évolution des pratiques alimentaires entre le Bronze ancien et La Tène finale est perceptible. En effet, si, pendant l'ensemble de la Protohistoire, la patelle et la scrobiculaire Scrobicularia plana sont consommées, la moule commune, l'huître plate Ostrea edulis et la palourde européenne Ruditapes decussatus n'intègrent le régime alimentaire des populations quà partir de la fin de l'âge du Fer. À cette époque, un changement considérable se produit en Basse-Normandie et en Poitou-Charentes. Des coquillages marins frais sont importés dans l'arrière-pays pour y être consommés, et ce jusqu'à $120 \mathrm{~km}$ du littoral. La consommation des coquillages dans les terres pouvait, à en juger par leur rareté, être réservée à quelques individus ou groupes d'un rang social élevé. Des réseaux d'échanges, voire un commerce des mollusques et plus largement des produits marins existaient probablement afin d'approvisionner ces sites continentaux.

Outre leur place dans l'alimentation, les invertébrés marins ont également joué un rôle dans plusieurs activités artisanales durant la Protohistoire. L'utilisation du pourpre dans des activités tinctoriales est attestée uniquement en Bretagne et ce au moins dès l'âge du Fer voire dès l'âge du Bronze, ce qui est, dans les deux cas, une information nouvelle pour l'ouest de la Gaule. En effet, cette activité n'était attestée jusqu'à présent sur tout le territoire français qu'après la conquête romaine. Par ailleurs, quatre espèces au moins de coquilles ont servi de matière première pour la parure, à savoir le cyprée Trivia 
monacha, la littorine obtuse Littorina obtusata, le dentale Antalis sp. et la coque Cerastoderma sp. La collection étudiée se caractérise par son hétérogénéité, autant d'un point de vue géographique, chronologique et contextuel que morphologique. Une différenciation marquée entre les espèces réservées à la parure et celles destinées à la consommation est observable pendant la Protohistoire, constat déjà réalisé par Catherine Dupont pour le Mésolithique et le Néolithique. Globalement, il semblerait qu'à l'âge du Bronze la coquille pour la confection de la parure soit remplacée progressivement par les métaux, matériaux plus résistants et permettant de créer des formes plus complexes. Les restes d'invertébrés marins et particulièrement les coquilles de mollusques sont parfois utilisés aussi comme matériaux de construction. Sur la façade atlantique française, le recyclage de coquilles en tant que matériaux de construction dans la construction des murs et pour l'épandage sur le sol concerne deux taxons, à savoir la patelle et l'huitre plate. La réutilisation de ces deux espèces est due à leurs propriétés physico-chimiques : leurs coquilles résistent aux pressions mécaniques, drainent les flux d'eau et sont perméables, absorbant l'humidité ambiante, souvent importante en milieu côtier et insulaire. L'utilisation des coquilles dans les constructions est proportionnellement liée à l'importance de leur consommation.

Enfin, les invertébrés marins jouaient également un rôle non négligeable au sein des systèmes de pen- sée et de croyances des populations protohistoriques. Ils sont ainsi parfois repérés sous forme de dépôts votifs, d'offrandes alimentaires et de reliefs de repas rituels ou communautaires. Ils ont été déposés, voire mis en scène, dans au moins trois secteurs sur la façade française de la Manche et de l'Atlantique : dans la plaine de Caen, sur les côtes bretonnes et en Charente-Maritime. Les espèces d'invertébrés marins intervenant dans les pratiques funéraires et cultuelles varient. Il s'agit le plus souvent de taxons consommés de manière régulière et faisant partie du régime alimentaire, comme la patelle en Bretagne ou la moule dans la plaine de Caen. Les coquillages de la famille des cardiidés (coque et bucarde Acanthocardia sp.) semblent également sélectionnés pour les événements particuliers liés aux pratiques cultuelles dans la plaine de Caen. Chaque contexte se caractérise par un assemblage spécifique, que ce soit au niveau des espèces choisies ou des objets associés. Il est ainsi difficile de différencier ces pratiques, qui correspondent à des manières de faire et de penser distinctes. Les sites étudiés sont également trop rares pour en déduire des généralités.

L'étude des restes coquilliers marins pendant la Protohistoire n'en est qu'à ses débuts, et les premières données obtenues sont prometteuses et innovantes. Les résultats soulignent ainsi les implications de ces animaux marins dans de nombreux domaines de la vie des communautés de lâge du Bronze et de lâge du Fer et permettent d'aborder des thématiques inédites.
2 SEPTEMBRE 2015

Université de Leyde

Membres du Jury

Directeurs de thèse

Corinne L. Hofman

Professeure, université de Leyde

Arie Boomert

Chercheur, université de Leyde

Rapporteurs

Maarten E.R.G.N. Jansen

Professeur, université de Leyde

Eduardo Góes Neves

Professeur, Musée d'archéologie et

d'ethnologie de l'université de São Paulo

Eithne B. Carlin

Chercheuse, université de Leyde

Examinateurs

Humphrey H. H. Kanhai

Professeur, université de Leyde

David R. Fontijn

Professeur, université de Leyde

Harry Fokkens

Professeur, université de Leyde

Stéphen Rostain

Directeur de recherche, CNRS

Andrzej T. Antczak

Chercheur, université de Leyde

Consultable

https://openaccess. leidenuniv.nl/ handle/1887/35054
Martijn M. van den Bel

Inrap

\section{Archaeological investigations between Cayenne Island and the Maroni river: a cultural sequence of western coastal Franche Guiana from $5000 \mathrm{BP}$ to present}

En Guyane française, la prescription de recherches archéologiques ne date que du début des années 1970. Elles ont été menées principalement sur le littoral qui s'étend entre 5 et $50 \mathrm{~km}$ depuis la côte atlantique vers l'intérieur des terres. Les connaissances, essentiellement corrélées au développement des infrastructures modernes, s'en trouvent biaisées et concernent surtout lî̂le de Cayenne et la plaine holocène pour l'Âge céramique tardif (AD 900-1500). Cette étude présente les résultats de six fouilles archéologiques préventives afin d'étoffer nos connaissances sur ce département. Au même titre, de nouvelles données sur l’Âge archaïque et céramique ancien, une période méconnue, sont présentées. Elles viennent combler un hiatus chronologique. L'étude propose également de nouvelles perspectives et pistes pour l'Âge céramique tardif concernant les pratiques funéraires, les complexes céramiques et l'alimentation des Amérindiens.

Après la présentation des problématiques, replacées dans le contexte historique, archéologique et géologique de la Guyane et du Surinam, les résultats de chaque fouille sont exposés par ordre chronologique. En premier lieu, le site Eva 2, qui se trouve en limite de la savane pléistocène de Malmanoury, entre Kourou et Sinnamary. Ce site, d'origine précéramique, a livré de la céramique ancienne ainsi que des pierres polies, du débitage sur enclume de quartz et des fours en cuvette à blocs de pierre. Les analyses d'amidon réalisées sur plusieurs meules ont démontré la préparation de patates douces et du maïs à partir de 2500 BC. L'ensemble du mobilier lithique du site Eva 2 correspond à la Tradition littorale et archaïque du nord de l'Amérique du Sud. On avance l'hypothèse que la présence de la céramique ancienne à partir d'environ $2200 \mathrm{BC}$ correspond à un changement en ce qui concerne la préparation de la nourriture, désormais cuite, dans des récipients en céramique, à la vapeur dans les fours. L'ensemble céramique a été défini en tant que complexe céramique de Balaté et attribué à l'Âge céramique ancien (Phase A), contemporain d'autres complexes céramiques, comme ceux du Guyana (Phase Alaka) et du Pará, au Brésil (Tradition Mina).

Des céramiques contemporaines ont été également trouvées pendant la première occupation du 